\title{
Quilotórax traumático y su resolución quirúrgica, una entidad poco frecuente. A propósito de un caso
}

\author{
Juan Carlos Vázquez-Minero,凶 Steve Arciniega-Belmont, Edgar Meza-Félix, \\ Adriana Hernández-García, Víctor Flores-Huerta, Rodrigo Morales-Villalobos, Demian Trueba-Lozano
}

Centro de Trauma de la Cruz Roja Mexicana, Ciudad de México, México.

RESUMEN. El quilotórax se define como la acumulación de quilo en la cavidad pleural. La etiología traumática no iatrogénica ocurre en 0.2 a $3 \%$ de todos los casos. Presentamos el caso de un hombre de 18 años de edad que ingresó con historial de agresión por terceras personas con instrumento punzocortante, la cual motivó toracotomía anterolateral derecha inicial para hemostasia de lesión pulmonar y rafia de lesión diafragmática en el seno posterior paravertebral derecho. En el posoperatorio se documentó gasto elevado a través de sonda endopleural compatible con quilotórax, que se corroboró mediante citoquímico con triglicéridos de $243 \mathrm{mg} / \mathrm{dl}$. Se intentó manejo conservador con ayuno y nutrición parenteral; sin embargo, al quinto día con estas medidas persistió con gasto superior a $1,000 \mathrm{~cm}^{3}$ e imagen radiográfica con cavidad y acumulación de líquido; por lo tanto, se llevó a cabo manejo quirúrgico con pleurodesis. El paciente tuvo adecuada evolución. Cinco días después inició, dieta sin datos de nuevo quilotórax y se efectuó retiro de drenaje pleural y egresó al séptimo día.

Palabras clave: Quilotórax, trauma torácico, trauma penetrante.

\section{Traumatic chylothorax and its surgical resolution, a rare entity. A case report}

ABSTRACT. Chylothorax is defined as the accumulation of chyle in the pleural cavity. The non-iatrogenic traumatic etiology occurs in 0.2 to $3 \%$ of all cases. We introduce the case of an 18-year-old male who was admitted with history of aggression with a sharp-pointed instrument, which resulted in initial right anterolateral thoracotomy with pulmonary injury hemostasis and right posterior paravertebral diaphragmatic sinus rhaphy. During the PO period, the chest-tube developed high output of chyle-like fluid. The diagnosis was corroborated with cytochemical study demonstrating triglycerides $243 \mathrm{mg} / \mathrm{dl}$. Conservative management was attempted with fasting and parenteral nutrition, but the output remained on $1,000 \mathrm{~cm}^{3}$ on the fifth post operatory day with imaging showing cavity and fluid accumulation despite these measures. Surgical management with pleurodesis was performed. The patient had an adequate evolution; on the five post operatory day the patient started oral nutrition without new chylothorax evidence, the chest-drainage was removed and the patient discharged on seven day.

Key words: Chylothorax, thoracic trauma, penetrating trauma.

\section{INTRODUCCIÓN}

Un paciente sano produce aproximadamente dos litros de líquido quiloso proveniente del tracto gastrointestinal en 24 horas, los cuales son trasportados a través el conducto torácico hacia el sistema vascular. Cuando ocurre una lesión de este conducto por cualquier mecanismo, sobreviene una rápida acumulación de quilo en el espacio pleural. ${ }^{1}$

\footnotetext{
$\triangle$ Autor para correspondencia:

Dr. Juan Carlos Vázquez-Minero, Centro de Trauma de Cruz Roja

Mexicana, Ciudad de México, México.

Correo electrónico: minerojc10@gmail.com
}

Trabajo recibido: 11-VI-2019; aceptado: 18-VI-2019
El quilotórax se define clínicamente como la presencia anormal de líquido de aspecto lechoso en el espacio pleural que se origina en el sistema linfático gastrointestinal. ${ }^{2,3}$ La primera descripción de un quilotórax traumático fue realizada por Langelot en el año de $1663 .^{4}$

El quilotórax se clasifica como no traumático y traumático. Cuando la etiología es traumática, el quilotórax se puede subclasificar como iatrógeno y no iatrógeno; la frecuencia de este último representa 0.2 a $3 \%$ de todos los casos. 5,6

El tratamiento clásico inicial de esta patología es conservador mediante ayuno y nutrición parenteral total, ya que en más de $50 \%$ de los casos ocurre cierre espontáneo sólo con estas medidas. ${ }^{7}$ Las indicaciones para optar por un manejo quirúrgico son: gasto a través

Este artículo puede ser consultado en versión completa en: www.medigraphic.com/neumologia 


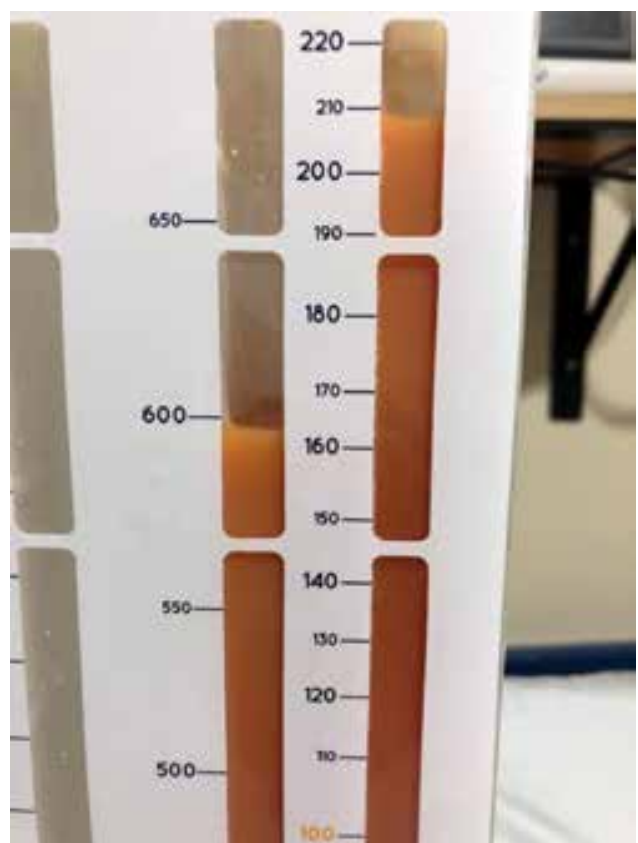

Figura 1: Líquido quiloso en el pleurovac.

de la sonda endopleural mayor de 1.5 I/día en adultos o mayor de $100 \mathrm{ml} / \mathrm{kg} /$ día en niños; gasto mayor de 1 l/día durante cinco días o salida de quilo persistente durante más de dos semanas. ${ }^{8}$

A continuación, presentamos el caso de un paciente con herida por arma punzocortante en quien se demostró quilotórax que no respondió a manejo conservador.

\section{PRESENTACIÓN DE CASO}

Hombre de 18 años de edad, quien ingresó con historial de agresión por terceras personas con instrumento punzocortante, con múltiples heridas en abdomen y tórax anterior y posterior. Fue recibido en cubículo de choque con datos clínicos francos de inestabilidad hemodinámica y síndrome de derrame pleural derecho, por lo cual se insertó sonda pleural con obtención de $1,200 \mathrm{~cm}^{3}$ de sangre. Se decidió llevar a cabo toracotomía anterolateral derecha; durante la misma, se efectuaron hemostasia de lesión pulmonar y rafia de lesión diafragmática de $3 \mathrm{~cm}$ en el ángulo posterior, cerca de la columna vertebral.

El paciente egresó a terapia intensiva con apoyos ventilatorio mecánico y aminérgico, manteniéndose relativamente estable; sin embargo, 18 h después de la intervención ocurrió sangrado a través del drenaje pleural de $1,000 \mathrm{~cm}^{3}$ asociado con caída de los parámetros hemodinámicos; se decidió, entonces, llevar a cabo reexploración quirúrgica, encontrando sangrado proveniente de la décima arteria intercostal que ameritó hemostasia mediante ligadura. El paciente reingresó en terapia intensiva y tuvo franca mejoría; no obstante, el gasto a través de la sonda pleural se mantuvo entre 1,500 y $2,000 \mathrm{~cm}^{3} /$ día de líquido seroso, el cual adquirió características quilosas al iniciar la administración de alimentos por vía oral (figura 1).

La impresión diagnóstica de quilotórax se corroboró mediante estudio citoquímico del líquido pleural, en donde se encontró color naranja, aspecto turbio, $\mathrm{pH} 8$, eritrocitos 50-60 por campo, leucocitos 4-6 por campo, polimorfonucleares $62 \%$, no se observaron bacterias, proteínas totales $2.0 \mathrm{~g} / \mathrm{dl}$, DHL $704 \mathrm{U} / \mathrm{L}$, glucosa $73 \mathrm{mg} /$ dl y triglicéridos $243 \mathrm{mg} / \mathrm{dl}$. Se realizó cultivo, que fue negativo a las $72 \mathrm{~h}$. Una vez diagnosticado, se decidió llevar a cabo manejo conservador mediante ayuno y nutrición parenteral total; no obstante, cuatro días después de establecer el tratamiento, el gasto a través del drenaje pleural persistió entre 600 y $800 \mathrm{~cm}^{3} /$ día con evidencia radiológica de acumulación de líquido en el espacio pleural (figura 2). Bajo estas aristas, se decidió llevar a cabo toracotomía posterolateral derecha, realizando drenaje del líquido acumulado, liberación de adherencias pulmonares y ligadura del tejido adiposo paravertebral, así como pleurodesis con iodopovidona. Veinticuatro horas después, se documentó gasto de 200 $\mathrm{cm}^{3}$ de material seroso a través de la sonda. Ocurrieron mejorías clínica y radiológica tres días después del evento quirúrgico (figura 3), por lo que se retiraron los drenajes al quinto día. Con tolerancia a dieta normal, egresó del servicio al séptimo día. Se llevó a cabo

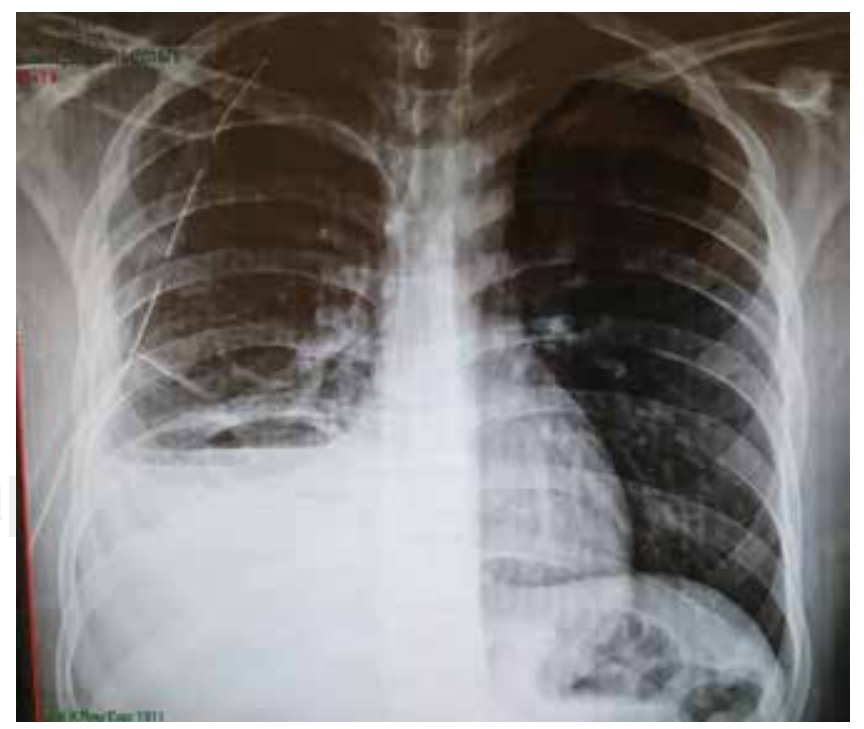

Figura 2: Derrame pleural derecho a pesar del drenaje torácico. 


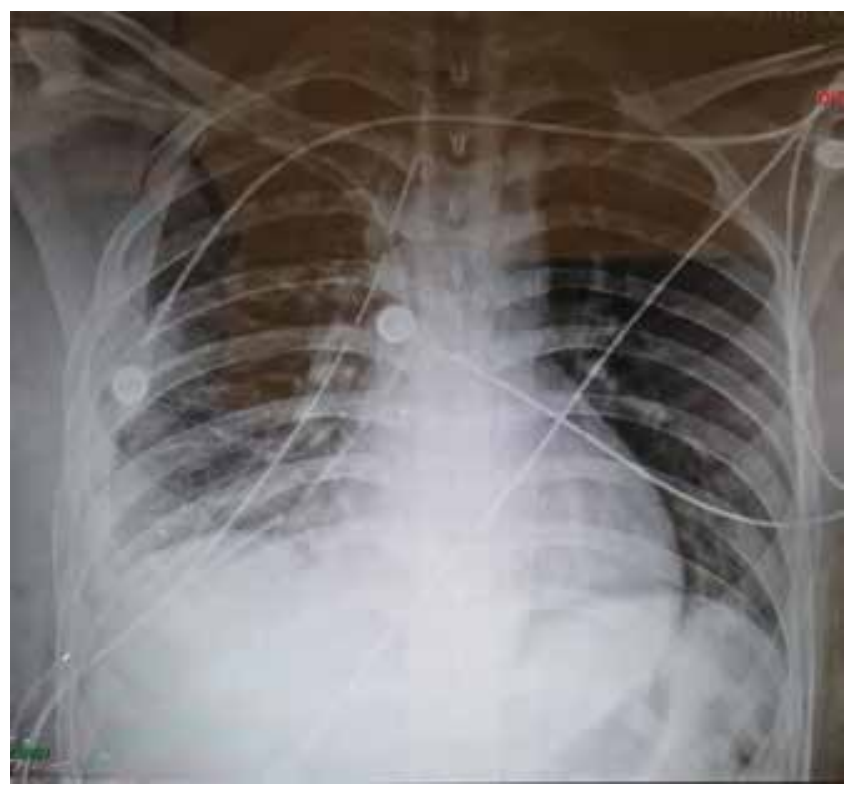

Figura 3: Radiografía posoperatoria a las 24 horas.

control médico un mes después de la intervención y se encontró con adecuadas evoluciones clínica y radiológica (figura 4).

\section{DISCUSIÓN}

Los casos de quilotórax traumático no iatrógeno reportados en la literatura son pocos, se efectuó una revisión de la literatura de los últimos cinco años en PubMed con el MESH «quilotórax postraumático», obteniendo un total de 32 artículos, donde sólo nueve correspondieron a esta categoría.

El quilotórax traumático comúnmente es de etiología iatrogénica: $80 \%$ por lesión del conducto torácico durante intervenciones como esofagectomía o neumonectomía. ${ }^{9}$ En la literatura se menciona que la causa del quilotórax hasta en un $51 \%$ es de etiología posquirúrgica; aunque en otros estudios se menciona que la causa infecciosa y el cáncer son las más frecuentes. En otros estudios se menciona que hasta un $60 \%$ es de causa no traumática, siendo la más frecuente las neoplasias malignas. Nuestro caso corresponde a una etiológica traumática no quirúrgica o no iatrógena, en donde el porcentaje es aún más bajo. ${ }^{10} \mathrm{El}$ quilotórax traumático no iatrógeno es una entidad poco frecuente (0.2 al 3\%); en nuestro hospital, sólo se habían presentado otros dos casos en un período de 20 años. ${ }^{5,6}$

Esta entidad puede ocurrir como consecuencia de trauma contuso asociado con fracturas de cuerpos vertebrales en $20 \%$ de los casos. Las lesiones por proyectil de arma de fuego y las lesiones por instrumentos punzocortantes — como en el paciente descrito- son más frecuentes. ${ }^{3}$

En cuanto al abordaje diagnóstico, la sospecha clínica es importante; sin embargo, sólo $50 \%$ de los casos se presenta con salida de líquido de características lechosas, en el resto puede tratarse de material seroso, serohemático, amarillo, verde o francamente hemático. En nuestro paciente, los gastos iniciales fueron serosos, incrementados en cantidad y sólo después de iniciar la administración de alimentos por vía oral, se evidenció la presencia de material característicamente quiloso. Por estos motivos, es necesario considerar la presencia de empiema como parte del diagnóstico diferencial. ${ }^{8}$

El diagnóstico definitivo se lleva a cabo mediante análisis del líquido pleural, caracterizado por la presencia de linfocitos y contenido de triglicéridos mayor de $110 \mathrm{mg} / \mathrm{dl}$, como ocurrió en el caso presentado. La presencia de quilomicrones o colesterol $\geq 200 \mathrm{mg} / \mathrm{dl}$ también es diagnóstica. ${ }^{8}$

El éxito general del tratamiento conservador oscila entre 20 y $80 \%$. El quilotórax traumático se resuelve en $50 \%$ de los casos mediante tratamiento conservador consistente en sonda pleural, ayuno por 14 días, nutrición parenteral total o triglicéridos de cadena media por vía oral —pues su absorción ocurre por vía portal-, pleurodesis y análogos de somatostatina. Si el gasto a través del drenaje es mayor a 1 It día durante cinco días consecutivos, persiste más de dos semanas o hay compromiso metabólico-nutricional, la cirugía suele ser el tratamiento de elección con ligadura del conducto torácico, pleurodesis química y pleurectomía (decorticación) o shunt pleuroperitoneal. ${ }^{7}$

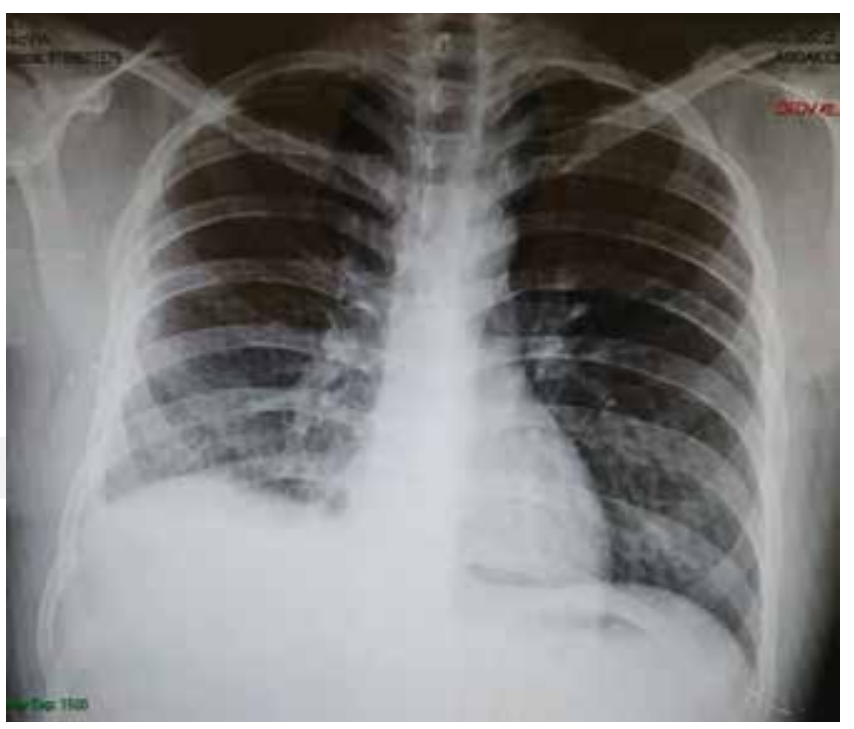

Figura 4: Radiografía de tórax al mes de operado. 
A pesar de estos criterios, la elección del mejor momento para optar por un manejo quirúrgico continúa siendo tema de debate, aunque los beneficios de una intervención temprana están claramente documentados y el éxito de la ligadura del conducto torácico se ha reportado en $90 \%$. Existen algunos avances como la embolización del conducto con altos porcentajes, según la literatura de éxito, estos procedimientos en la mayoría de los hospitales no están disponibles; por lo tanto, no superan el manejo médico o quirúrgico convencional, en nuestro centro hospitalario no existen esta posibilidad terapéutica.

Las intervenciones toracoscópicas son alternativas atractivas para el abordaje por toracotomía habitual, pues disminuyen el trauma quirúrgico, aunque en pacientes posoperados, como en este caso, no son una alternativa adecuada, tal vez más útiles en etiologías no traumáticas. En nuestro paciente, el manejo inicial con nutrición parenteral total y ayuno por vía oral redujo el gasto a través del drenaje de 2,000 a $800 \mathrm{~cm}^{3}$ en 24 horas. Sin embargo, la radiografía no demostró mejoría; por el contrario, evidenció aumento del nivel de líquido y falta de expansión pulmonar a pesar de la sonda pleural. Ante esta situación, fue mejor el manejo quirúrgico con el escenario de dos cirugías torácicas previas, teniendo como objetivo el drenaje del líquido, la liberación y expansión pulmonar, la pleurodesis y la ligadura de la grasa paraórtica. ${ }^{8,10}$

En los quilotórax traumáticos, el tratamiento inicial de elección es conservador, dado que hasta en $50 \%$ de los casos el conducto torácico se cierra espontáneamente en las dos primeras semanas. ${ }^{3}$ Otros autores mencionan el éxito en el tratamiento hasta del 100\% de los casos, cuando la etiología es no traumática, en particular neoplásica, con la realización de pleurodesis mediante toracoscopia. ${ }^{11}$

El tratamiento que muchos estudios recomiendan en el caso de lesiones por trauma penetrante es la ligadura del conducto torácico, con lo cual la mejoría del paciente en este caso, al ligar la grasa paravertebral, probablemente se ligó alguna rama del conducto torácico; por eso, la mejoría notoria al día siguiente del procedimiento. Muchos hospitales no cuentan con las alternativas en boga debido a sus costos elevados o la falta de personal médico que pueda llevar a cabo las intervenciones. Así, la toracotomía con ligadura del conducto y pleurodesis sigue siendo un tratamiento de elección. ${ }^{12}$

\section{CONCLUSIONES}

El quilotórax postraumático no iatrógeno por mecanismo penetrante es una patología poco frecuente. En la mayoría de los casos suele evolucionar de manera satisfacto- ria mediante tratamiento conservador y, en la actualidad, se sugiere ofrecer tratamientos poco invasivos. La toma de decisión con respecto de operarlo o no debe basarse tanto en la falta de respuesta al manejo no operatorio, la observación radiológica secuencial y la disponibilidad de recursos. La acumulación persistente de líquido a pesar de los drenajes pleurales adecuadamente insertados debe ser considerada una de las indicaciones para explorar la cavidad e intentar resolver la causa del problema. El seguimiento estrecho de este tipo de pacientes y la decisión oportuna para llevar a cabo una cirugía son los factores decisivos para un buen resultado.

\section{Conflicto de intereses}

Los autores declaran no tener conflicto de intereses

\section{REFERENCIAS}

1. Macfarlane JR, Holman CW. Chylothorax. Am Rev Respir Dis 1972;105(2):287-291.

2. Hillerdal G. Chylothorax and pseudochylothorax. Eur Respir J 1997;10(5):1157-1162.

3. Carrillo-Esper R, Sosa-García JO, Carrillo-Cordova LD. Quilotórax secundario a herida por proyectil de arma de fuego. Cir Ciruj 2009;77(6):479-482.

4. Baldridge RR, Lewis RV. Traumatic chylothorax: $A$ review of the literature and report of a case treated by ligation of the thoracic duct and cisterna chyli. Ann Surg 1948;128(6):1056-1078.

5. Ikonomidis JS, Boulanger BR, Brenneman FD. Chylothorax after blunt chest trauma: a report of 2 cases. Can J Surg 1997;40(2):135-138.

6. López-Espadas F, Iribarren-Sarrías JL, Martínez-Jiménez C, Fernández-Rico R, Lacruz-Cañas A, Quesada-Suescun A. Quilotórax secundario a traumatismo torácico cerrado. A propósito de 6 casos. Arch Bronconeumol 1997;33(4):168-171.

7. Buettiker V, Hug MI, Burger R, Baenziger O. Somatostatin: a new therapeutic option for the treatment of chylothorax. Intensive Care Med 2001;27(6):1083-1086.

8. McGrath EE, Blades Z, Anderson PB. Chylothorax: aetiology, diagnosis and therapeutic options. Respir Med 2010;104(1):1-8. doi: 10.1016/j.rmed.2009.08.010.

9. Sendama W, Shipley M. Traumatic chylothorax: A case report and review. Respir Med Case Rep 2015;14:47-48. doi: 10.1016/j.rmcr.2015.01.001.

10. Martínez-Díaz JL, Torre-Bouscoulet L, Cortés-Télles A, et al. Quilotórax en pacientes con derrame pleural en el Instituto Nacional de Enfermedades Respiratorias, 2011-2012. Neumol Cir Torax 2013;72(3):207-211.

11. Pillay TG, Singh B. A review of traumatic chylothorax. Injury 2016;47(3):545-550. doi: 10.1016/j.injury.2015.12.015.

12. Cortés-Télles A, Rojas-Serrano J, Torre-Bouscoulet L. Quilotórax: frecuencia, causas y desenlaces. Neumol Cir Torax 2010;69(3):157-162. 